\title{
Improved in-situ structural evaluation of historic buildings incorporating a 3D laser scanning technique
}

\author{
C.-J. Chen ${ }^{1} \&$ T.-C. $\mathrm{Wu}^{2}$ \\ ${ }^{I}$ Graduate Institute of Urban Development and Architecture, \\ National University of Kaohsiung, Taiwan \\ ${ }^{2}$ Department of Water and Soil Conservation, \\ National Chung Hsing University, Taiwan
}

\begin{abstract}
A large percentage of historic buildings in Taiwan are of the traditional construction called Chuan-Dou, which consists of posts and mortised beam elements. The walls are usually filled with wattle and daub, and brick or wood planks. Experience has shown that these historic buildings perform moderately well from a strength perspective, but perform with less than good ductility under earthquake waves. Many buildings have collapsed mainly due to material degradation and structural instability. In order to conserve this kind of building, in-situ structural evaluations should be conducted before any renovation or any intervention is done, especially in a seismic-prone area like Taiwan. In general, conventional structural evaluation is based on the analysis of a simplified structural model (i.e., the 2D idealized structural models according to the in-situ survey and drawings). Without considering 3D effects as well as real geometric conditions of structure (e.g., deformation, inclination, etc.), the structural characteristics such as rotational stiffness, displacements, and stress of members are usually underestimated or overestimated. This consequently results in the misjudged restoration or inefficient reinforcement in further interventions. This study proposes a new method incorporating a 3D laser scanning technique, which allows the recording of the real structural geometric conditions, which can be simultaneously converted into 3D models for structural analysis. This improved method increases not only the efficiency of structural evaluation, but also conforms more precisely to the real state of the structure. This paper compares the efficiency and accuracy of structural evaluations between the conventional and the new methods. The measuring technique and the procedure of structural analysis are discussed.
\end{abstract}

Keywords: historic building, laser scanning, structural evaluation. 


\section{Introduction}

The structural evaluation is one of the most critical stages before the conservation plans are defined. Based on the results from in-situ investigations and a series of studies, the material quality and the component structural functions are evaluated. Some structural modeling will be undertaken to ensure that the exact structural behavior is obtained for the integrity of the structure. The precision of such analysis is usually influenced by the accuracy of in-situ investigations, especially the geometric survey and mapping. The established drawing, which is developed at a later stage, will provide the basis for structural models. In general, the 2D idealized and simplified models are used without considering the 3D effect and the real geometric conditions of structure (i.e., deformation and inclination). A more realistic analysis can be derived and some indistinct estimations of structural intervention can thus be avoided.

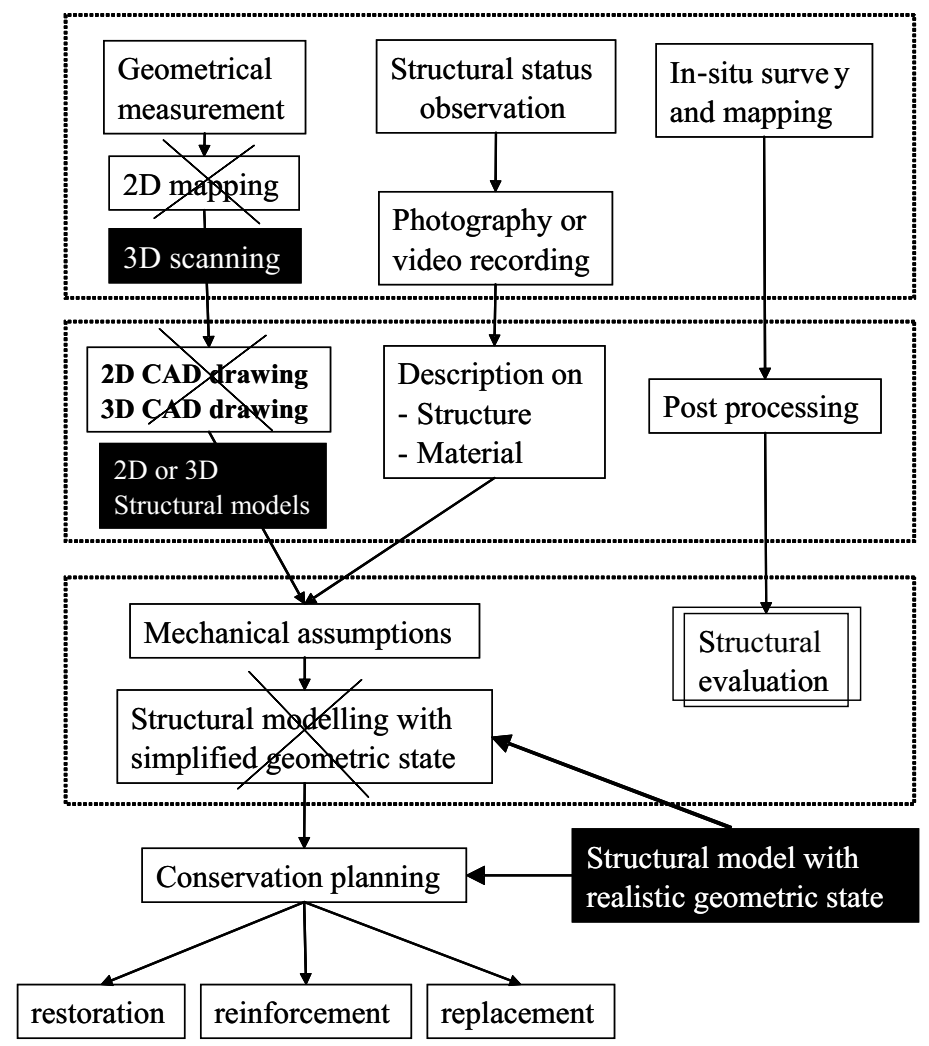

Figure 1: Conventional process of structural evaluation and its modification. 


\section{Structural evaluation process}

\subsection{Conventional process}

Figure 1 shows the conventional process, which uses the traditional observationrecoding-drawing-modeling process. The deficiency of this method is that the final mapping and drawing assume usually the existing structure is intact geometrically and remains stable in appearance. Some underestimation or overestimation will lead to an inaccurate conservation plan later on.

\subsection{Proposed solution}

The proposed solution is to use the 3D laser scanning technique instead of a more traditional method. The new process will provide accurate and rapid observation and recoding. The registered data can then be converted into a realistic 3D model; with the simplified 2D diagram for the modeling as well. Figure 1 indicates that the use of 3D laser scanning can replace rough drawing and can minimize processing time constraints by jumping from the in-situ survey stage to the post processing stage. Both the time saved and the accuracy of the process can be increased in such a working procedure.

\section{3D laser scanning technique}

3D laser scanning technique is applied more frequently in projects for historical building conservation. This technique can capture physical objects such as structures or scenes and converts them into digital point cloud data for follow-on processing. The appropriate software program processes direct point cloud data into 3D models. The user can manipulate this model, extract its geometry, or create surface models and output the data to simple or complicated models. The technique is ideal for in-situ investigation that needs to capture existing geometries and physical relationships. Compared to the conventional methods, such as traditional drawings, which are usually inadequate and imprecise or impractical to use, the 3D laser scanning technique can boost the efficiency and the precision of in-situ investigation.

\section{Case study and descriptions}

The studied case takes a traditional and typical Chuan-Dou building located at Tainan County in Southern Taiwan. The structural system of the building consists of a timber frame and different wall systems with wattle and daub and featuring brick and wood planks. All primary wooden components are mortised with different methods that are invisible. Figure 2 shows the exterior front view of building. Figure 3 shows the interior wooden frame. The 3D laser scanning technique is applied to measure this building in order to analyze the structure. Non-destructive testing is also used to define the type of joints for the mortised elements of structure. The different joint properties can be introduced based on these results of in-situ investigation. 
432 Structural Studies, Repairs and Maintenance of Heritage Architecture X

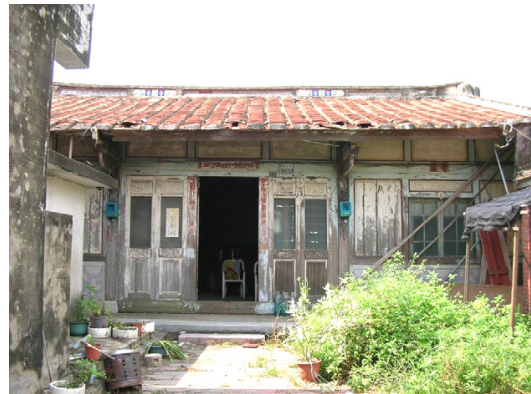

Figure 2: Exterior front view.

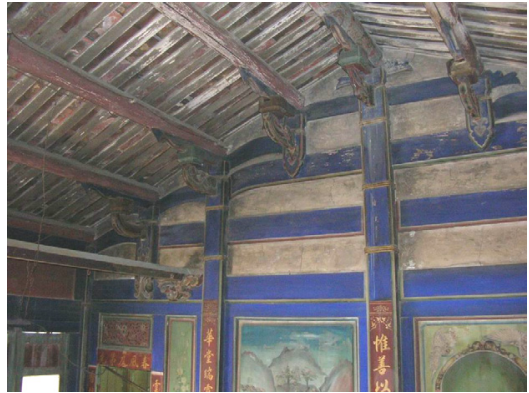

Figure 3: Interior view of frame.

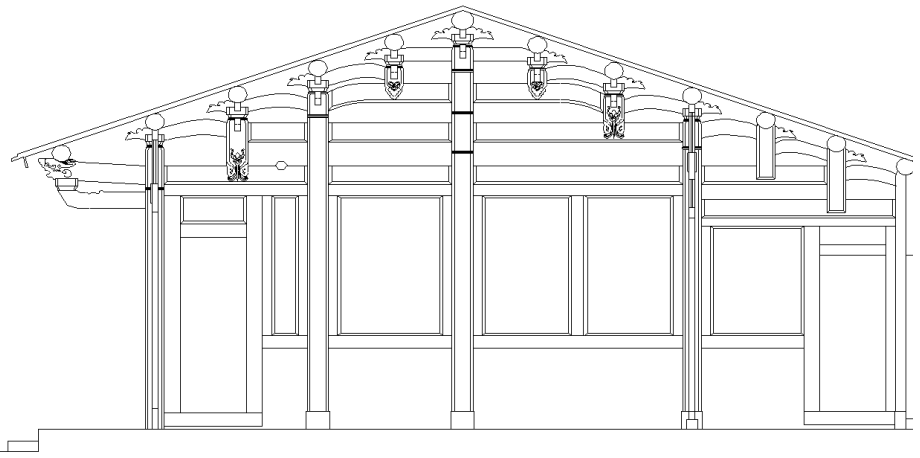

Figure 4: $\quad$ CAD drawing of structure.

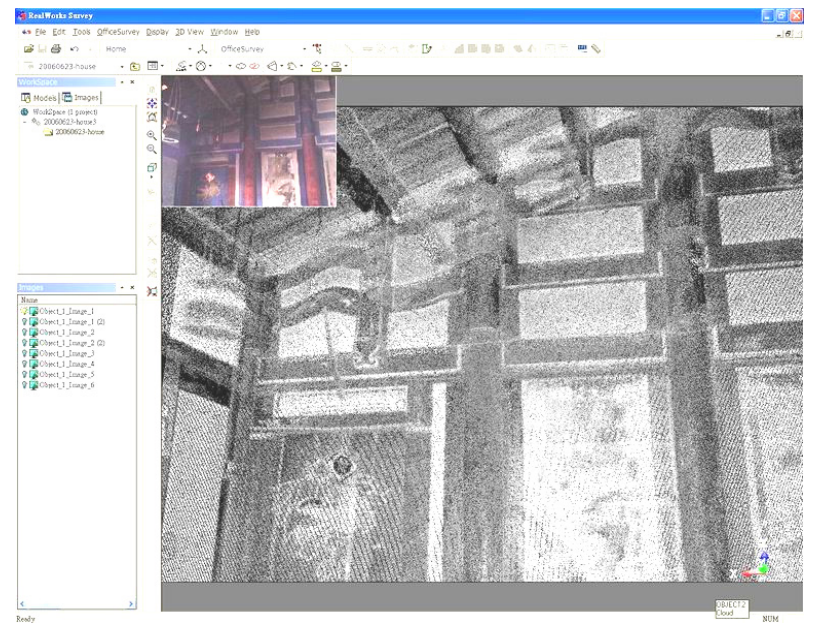

Figure 5: Scanned image of structure with point cloud. 

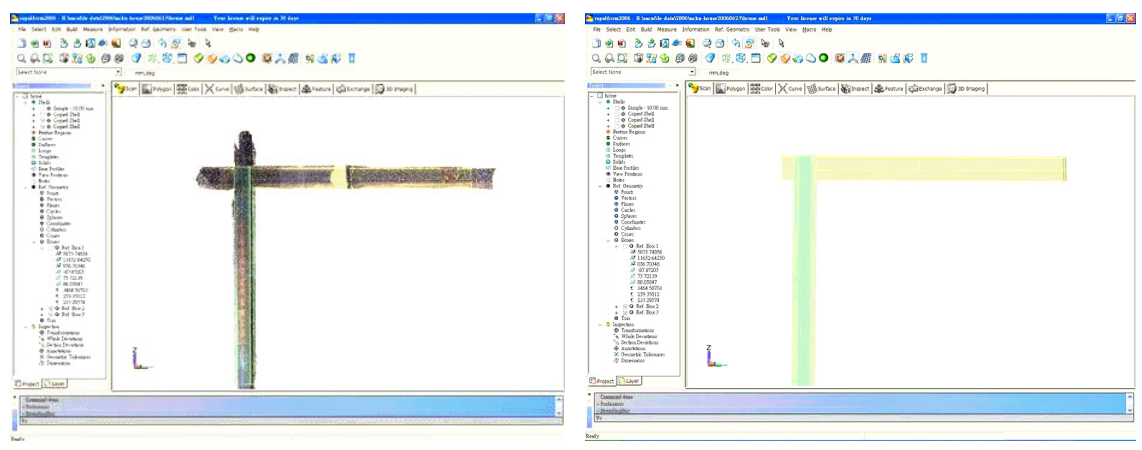

Figure 6: Transformation of digital image to geometrical data.

Figures 5 and 6 are the resulting images from 3D laser scanning. The scanned images can be transformed into simpler drawing data, which illustrates the exact geometrical state of investigated structure and offers a realistic structural model for further numerical analysis.

\section{Structural modeling}

The 3D structural model is established to analyze the stress distribution and the deformation of structure. SAP2000 V10 is chosen as the modeling platform where the linear static analysis is undertaken.

\subsection{Different assumptions}

In conventional structural modeling, the joints are assumed to be as rigid or hinged. In this study, the joints are defined as their real states of different joint types with continued, flat cut and dovetailed. The real rotational stiffness is introduced as spring stiffness into models as spring stiffness of joints. The deformation of existing structure is considered as the initial geometrical condition instead of considering that the structure is under an intact state. These different assumptions will provoke different stress distributions within the analyzed structures. This study compares the numerical results of two different assumptions: (1) the hinged joints and a semi-rigid joint, and (2) the idealized geometrical condition versus a realistic deformed state.

\subsection{Semi-rigid behavior}

It is well known that the joint behavior has a very large influence on the response of the whole structure (not regarding the material) for civil engineering, buildings, and structures. The semi-rigid behavior seems more realistic for most of the structural joints with different materials. A number of recent studies determined that the semi-rigidity, being defined as relative rotation between posts and beams, may significantly influence the integrity of structure. The 
beam-springs models are usually applied to simulate the semi-rigid behavior of the joints in structural frames with the following assumptions:

(1) The frame is moment-resistant, the axial and shear deformation are neglected.

(2) The flexural moments are linearly distributed and the flexural stiffness is uniformly distributed in each beam member.

The moment-rotational relationship can be expressed as the following matrix:

$$
\begin{aligned}
& \left\{\begin{array}{l}
\Delta \theta_{A} \\
\Delta \theta_{B}
\end{array}\right\}=\frac{L}{6 E I}\left[\begin{array}{cc}
2+\xi_{A} & -1 \\
-1 & 2+\xi_{B}
\end{array}\right]\left\{\begin{array}{l}
\Delta M_{A} \\
\Delta M_{B}
\end{array}\right\}=[f]\{\Delta M\} \\
& \xi_{A}=\frac{6 E I}{C_{A} L}, \xi_{B}=\frac{6 E I}{C_{B} L}
\end{aligned}
$$

where $C_{A}$ and $C_{B}$ are the spring stiffnesses, $[f]$ is the flexibility matrix of the member.

The inverse relationship of the previous equation can be written as:

$$
\left\{\begin{array}{l}
\Delta M_{A} \\
\Delta M_{B}
\end{array}\right\}=\frac{6 E I}{L\left(3+2\left(\xi_{A}+\xi_{B}\right)+\xi_{A} \xi_{B}\right.}\left[\begin{array}{cc}
2+\xi_{B} & -1 \\
-1 & 2+\xi_{A}
\end{array}\right]\left\{\begin{array}{l}
\Delta \theta_{A} \\
\Delta \theta_{B}
\end{array}\right\}=[K]\{\Delta \theta\}
$$

$[\mathrm{K}]$ is the stiffness matrix of the member.

\subsection{Joint assumption}

This study refers to the relative research (Chang, 2005) of the rotational stiffness by involving static linear analysis and experimental verifications. Different rotational stiffness for the three usual types of joints of the Chuan-duo style building is defined as follows:

(1) Continued joints

For the joint with mortise and continued beam, the relationship of momentrotation can be expressed as:

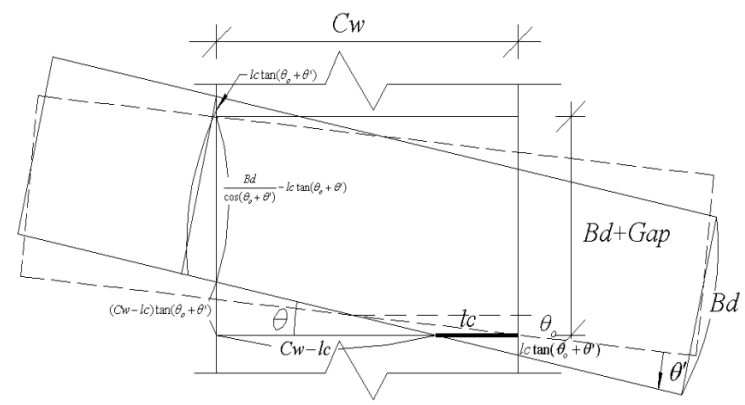

Figure 7: $\quad$ Rotated continued joint. 
Figure 7 shows the geometric state of a rotated continued joint.

$$
\begin{aligned}
M & =\left(C w-\frac{2}{3} l c\right) \cdot f(\theta) \\
& =\frac{3-2 \cdot \alpha(\theta)}{6} \cdot \frac{C w^{3} \cdot B w \cdot E_{\perp}}{B d} \cdot \alpha^{2}(\theta) \cdot \beta(\theta) \cdot \sin \theta
\end{aligned}
$$

where $C w$ is the width of the post, $B w$ is width of the beam, $B d$ is the depth of the beam, $E_{\perp}$ is the MOE perpendicular to wood grains, $\theta$ is the rotation angle.

(2) Flat cut joint

Figures 8 and 9 show the geometric state of a rotated joint.

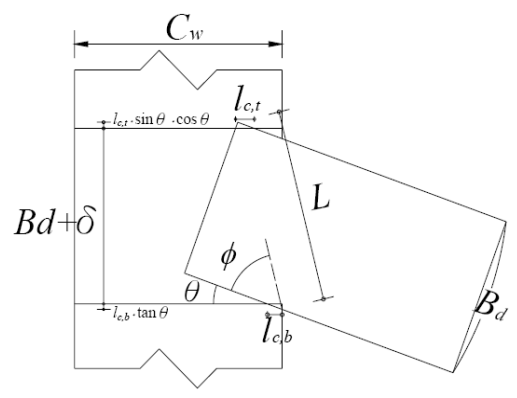

Figure 8: $\quad$ Rotated flat cut joint.

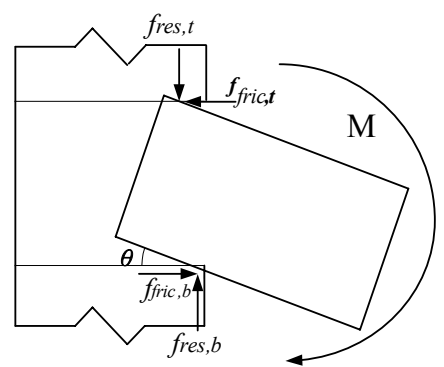

Figure 9: Frictions due to rotation of joint.

For the joint with a mortised and flat cue beam, the moment stiffness is the total effect of slide friction and embedded friction between the beam and the post while rotation is occurring. The relationship of moment-rotation can be written as follows:

$$
M_{e m}(\theta)=L_{e m}(\theta) \cdot f_{\text {res }, t}(\theta)
$$

where

$$
\begin{aligned}
L_{e m}(\theta) & =L \cdot \cos (\theta+\phi)-l_{c, t} \cdot\left(\frac{2}{3} \cdot \cos ^{2} \theta-\frac{1}{3}\right)-\frac{1}{3} \cdot l_{c, b} \\
& =L \cdot \cos (\theta+\phi)-\frac{1}{3} \cdot l_{c, t} \cdot\left[2 \cdot \cos ^{2} \theta+\cos \theta-1\right] \\
f_{r e s, t}(\theta) & =\frac{1}{2} \cdot l_{c, t} \cdot B_{w} \cdot \varepsilon_{\max , t} \cdot E_{\perp} \cdot \beta(\theta) \\
& =\frac{1}{2} \cdot l_{c, t}{ }^{2} \cdot \frac{B_{w} \cdot E_{\perp}}{B d} \cdot \beta(\theta) \cdot \sin \theta \cdot \cos ^{2} \theta
\end{aligned}
$$

where $C w$ is the width of the post, $B w$ is the width of the beam, $B d$ is the depth of the beam, $E_{\perp}$ is the MOE perpendicular to wood grains, $\theta$ is the rotation angle. 
Part of the moment-resistance provided from the friction, can be written as follows:

$$
\left\{\begin{array}{l}
f_{\text {fric }, t}(\theta)=\mu \cdot f_{\text {res }, t} \\
f_{\text {fric }, b}(\theta)=\mu \cdot f_{\text {res }, b}
\end{array}\right.
$$

where $\mu=0.4 \sim 0.6, f_{\text {res }}$ is the friction due to rotation of the joint.

The moment from slide friction is written as follows:

$$
M_{\text {fric }}(\theta)=L_{\text {fric }} \cdot f_{\text {fric }}(\theta)
$$

where

$$
L_{\text {fric }}=B d+\delta \text { 。 }
$$

(3) Dovetailed joint

For the joint with a mortised and dovetail beam, the relationship of momentrotation can be expressed as follows:

$$
M_{D C}=\left(0.3943 \times B w \cdot E_{\perp}-4.1887\right) \cdot\left(1-e^{-16.835 \times \theta}\right)
$$

The initial stiffness of this type of joint can be written as follows:

$$
K_{i, D C}=6.223 \times B w \times E_{\perp}-290.61
$$

where $B w$ is the width of the beam, $E_{\perp}$ is the MOE perpendicular to wood grains.

These different rotational stiffnesses are introduced as spring stiffnesses in numerical modeling.

\subsection{Basic properties of material and structure}

According to the different investigations of traditional Chuan-Dou style buildings, the mechanical properties can be summarized as follows:

- Material Chinese cypress (Cunninghamia lanceolata)

- Modulus of elasticity (parallel to wood grains): $7.0 \times 10^{8} \mathrm{~kg} / \mathrm{m}^{2}$

- Modulus of elasticity (perpendicular to wood grains): $2.8 \times 10^{7} \mathrm{~kg} / \mathrm{m}^{2}$

- Poisson's ratio: 0.4

- Density of wood: $360 \mathrm{~kg} / \mathrm{m}^{3}$

- Roof weight (dead load of tiles): $120 \mathrm{~kg} / \mathrm{m}^{2}$

The sections of the components are: $18 \mathrm{~cm} \times 18 \mathrm{~cm}$ for the middle post, $16 \mathrm{~cm} \times 16 \mathrm{~cm}$ for the other posts, and $9 \mathrm{~cm} \times 12 \mathrm{~cm}$ for the mortise beams and $10 \mathrm{~cm} \times 10 \mathrm{~cm}$ for the vertical short posts.

The load combination includes dead load of members, roof load and a lateral joint load, which induces the rotation effect between posts and beams. 


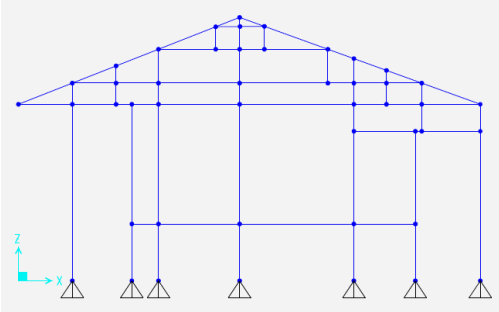

Figure 10: Numerical model.

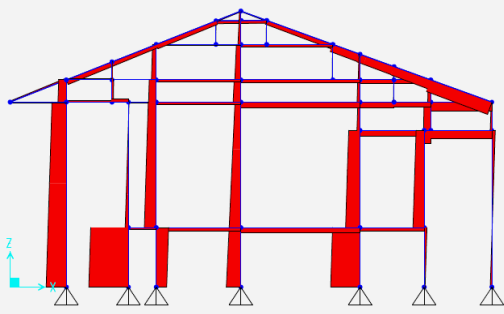

Figure 12: Axial forces of idealized structure.

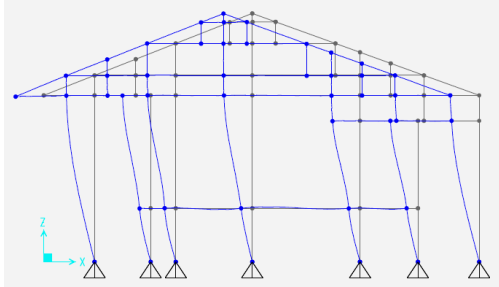

structural Figure 11: Deformation of structure.

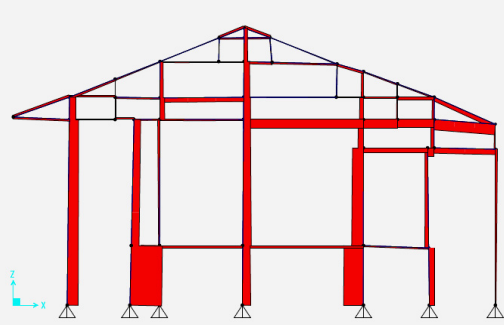

Figure 13: Axial forces of realistic structure.

Figures 10 and 11 are the numerical model using the software package SAP2000, before loading and after loading.

Table 1: $\quad$ Comparison of structural modelling.

\begin{tabular}{|c|c|c|c|}
\hline Models & $\begin{array}{c}\text { Horizontal } \\
\text { Displacement } \\
(\mathrm{cm})\end{array}$ & $\begin{array}{c}\text { Vertical } \\
\text { Displacement } \\
(\mathrm{cm})\end{array}$ & $\begin{array}{c}\text { Maximal Axial } \\
\text { Force (kgf) }\end{array}$ \\
\hline $\begin{array}{c}\text { Idealized model } \\
\text { with hinged joints }\end{array}$ & 0.551 & 0.644 & 26872 \\
\hline $\begin{array}{c}\text { Idealized model w. } \\
\text { semi-rigid joints }\end{array}$ & 0.537 & 0.329 & 28715 \\
\hline $\begin{array}{c}\text { Realistic model w. } \\
\text { semi-rigid joints }\end{array}$ & 1.298 & 2.16 & 48340 \\
\hline
\end{tabular}

\subsection{Comparison of structural modeling}

Table 1 shows the different results of structural modeling. For the middle top of the frame, the idealized model with semi-rigid joints behaves more rigidly compared with the others. The realistic model has the maximal displacements in both vertical and horizontal directions. For axial forces in posts, the maximal 
value is found in the realistic model with semi-rigid joints. It is logical that the hinged assumption leads to the structure performing in a more flexible manner compared with the semi-rigid joints. The realistic model with semi-rigid joints with initial displacements, which reflects geometrical inclination, shows more displacements as well as maximal axial forces in components.

\section{Conclusion}

The 3D laser scanning technique can improve the efficiency and accuracy of insitu investigation. Not only the time saving of the process from in-situ 2D mapping to $2 \mathrm{D}$ or $3 \mathrm{D} \mathrm{CAD}$ drawing can be expected, but also the exact geometrical condition of structure can be obtained. Besides, by combining the scanned data and the semi-rigid joints assumptions, the structural evaluation becomes more reliable using an exact structural model in numerical analysis. In the case study, the realistic assumption affects higher displacements and stresses of existing structure. The results obtained can consequently influence the design sizes of stressed components without underestimation. The exact stiffness and structural behavior of the whole structure can direct the precise engineering interventions of renovation.

\section{References}

[1] Chi-Jen Chen, "Structural evaluation and of Traditional Chuan-Dou Timber Structures and earthquake resistance", ABRI scientific report, 2006.

[2] Wen-Shao Chang, "On Rotational Performance of Traditional Chuan-Dou Timber Joints in Taiwan”, Doctoral thesis, National Cheng Kung University, Taiwan, 2005.

[3] Z. B. Petkov, T. V. Vassilev, G. K., Gospodinov "Numerical Analysis of Plane R/C Frames using Joint Test Data", Proceeding of the Second State of the Art Workshop, Prague 1994, pp. 541 546. 